\title{
Synchrotron radiation as a probe of confinement and QGP
}

\author{
V. V. Goloviznin \\ Bogolyubov Institute for Theoretical Physics, National Academy of Sciences of Ukraine, Kiev, \\ 03143, Ukraine \\ E-mail: goloviznine@netscape.net
}

\section{A. V. Nikolskii*}

Bogoliubov Laboratory of Theoretical Physics, JINR, 141980, Dubna, Russia

E-mail: alexn@theor.jinr.ru

\section{A. M. Snigirev}

Skobeltsyn Institute of Nuclear Physics, Lomonosov Moscow State University, 119991, Moscow,

Russia, and

Bogoliubov Laboratory of Theoretical Physics, JINR, 141980, Dubna, Russia

E-mail: Alexandre.Sniguirev@cern.ch

\section{G. M. Zinovjev}

Bogolyubov Institute for Theoretical Physics, National Academy of Sciences of Ukraine, Kiev, 03143, Ukraine

E-mail: Gennady.Zinovjev@cern.ch

\begin{abstract}
The intensive synchrotron radiation resulting from quarks interacting with the collective confining color field in heavy ion collisions is discussed. The spectrum of hard photons is presented and is compared with the experimental data showing a feasibility of this magnetic bremsstrahlung-like radiation. A study of the earlier predicted specific anisotropy in the angle distribution of dileptons with respect to the three-momentum of the pair is suggested as a way to select this mechanism among other sources of direct photons and dileptons and to probe the confinement properties.
\end{abstract}

International Conference on Hard and Electromagnetic Probes of High-Energy Nuclear Collisions 30 September - 5 October 2018

Aix-Les-Bains, Savoie, France

${ }^{*}$ Speaker. 


\section{Introduction}

Investigation of matter properties under extreme conditions, for example, quark-gluon plasma (QGP) is one of the most relevant and interesting problems of modern high-energy physics with heavy-ion collisions. Among the possible ways of studying the QGP are the so-called electromagnetic probes $[1,2,3]$, that is, photon and lepton sources. Since these particles can pass through the plasma volume, practically without interacting with quarks and gluons, they, therefore, carry direct information about its structure.

We propose an essentially alternative mechanism for possibly explaining the observed azimuthal anisotropy of direct photons with respect to the reaction plane, which apparently is not taken into account in other phenomenological approaches. We are talking about "magnetic bremsstrahlung" or synchrotron radiation of quarks in the collective color field, providing confinement.

This talk is mainly based on our studies $[4,5,6]$, which we summarize here.

\section{Theoretical framework}

In our approach, we assume that the interaction of quarks and QGP occurs by means of a constant holding force $\sigma$ that acts along the normal to the surface of the plasma volume. As a result, a colored object, i.e. a quark or antiquark at the boundary of the plasma system begins to move along a curved trajectory, so that it emits photons. This magnetic bremsstrahlung radiation is sufficiently intensive for light quarks due to the large magnitude of the "string tension" $\sigma \simeq$ $0.2 \mathrm{GeV}^{2}$ as fixed in the chromoelectric flux tube model [7, 8]. This effect is inherent to any accelerated moving classical charged particle. This quasi-classical treatment is obviously grounded on the fact that the de Broglie wavelengths of medium constituents are smaller being compared with the size of their localization region (QGP).

For the light $u$ - and $d$-quarks with the energy $E$ the probability of emitted photons with the frequency $\omega$ per unit time $t$ is independent of the mass of the emitting particle in the strong-field case and the spectral distribution can be represented as [9]:

$$
\frac{d N_{\gamma}}{d \omega d t}=\frac{1}{2} e_{q}^{2} \alpha \omega^{-2 / 3}(\sigma \sin \varphi / E)^{2 / 3}, \quad 0<\omega<E,
$$

where $\alpha=1 / 137$ is the fine structure constant, $e_{q}$ is the quark charge in units of electron charge and $\varphi$ is the angle between the quark velocity and the direction of quark confining force $\sigma$ (the normal to the QGP surface in our case). Knowing the laws of quarks motion, it is easy to determine the spectral angular distribution of photons radiated per unit surface area $S$ of plasma:

$$
\frac{d N_{\gamma}}{d S d t \omega^{2} d \omega d \Omega}=\frac{g\left\langle e_{q}^{2}\right\rangle \alpha}{(2 \pi)^{3} \sigma^{1 / 3}} \frac{3}{7} \omega^{2 / 3} \sin ^{2 / 3} \varphi_{0} \times \int_{1}^{\infty} d \xi \exp \left(-\frac{\omega}{T} \xi\right)\left(\xi^{7 / 3}-1\right),
$$

where $\left\langle e_{q}^{2}\right\rangle=e_{u}^{2}+e_{d}^{2}, \quad e_{u}$ and $e_{d}$ are the $u$ - and $d$-quark charges, $g=$ spin $\times$ color $=6$ is the number of quark degrees of freedom, $T$ is the plasma temperature, $\omega \xi=E$ is the initial quark energy. $\varphi_{0}$ is the angle between the normal to QGP surface and the direction of emitted photons. 
The basic conditions to have such a radiation available as: 1) confinement; 2) the presence of relativistic light quarks ( $u$ - and $d$-quarks) in the hot medium; 3 ) the semiclassical nature of their motion.

To specify the QGP geometry and evolution, we use a model of a cylindrical symmetric plasma volume expanding in longitudinal direction and use a hydrodynamical scaling solution. Taking for the QGP an ideal gas equations of state, we have $T=T_{0}\left(\tau_{0} / \tau\right)^{1 / 3}$, where $T_{0}$ is the temperature at the proper time $\tau_{0}$ at the beginning of the hydrodynamic expansion.

\section{The spectrum of synchrotron photons}

From our "master" Eq. (2.2) in the limit of large transverse momenta, $k_{\perp} \gg T$, we obtain:

$$
\frac{d N_{\gamma}}{d S d t k_{\perp} d k_{\perp}}=\Xi\left\langle e_{q}^{2}\right\rangle \alpha \sigma^{-1 / 3} k_{\perp}^{-5 / 6} T^{5 / 2} e^{-k_{\perp} / T}
$$

where $\Xi \simeq 0.29$ is the result of averaging over angles. Integration of Eq. (3.1) over the QGP surface, taking into account evolution, gives:

$$
\begin{aligned}
\frac{d^{2} N_{\gamma}}{2 \pi k_{\perp} d k_{\perp} d y}=\int \frac{d N_{\gamma}}{d S d t k_{\perp} d k_{\perp}} r \tau d \tau & = \\
& =\Xi\left\langle e_{q}^{2}\right\rangle \alpha 3\left(\tau_{0} T_{0}^{3}\right)^{2} r k_{\perp}^{-13 / 3} \sigma^{-1 / 3}\left[\Gamma\left(\frac{7}{2} ; \frac{k_{\perp}}{T_{0}}\right)-\Gamma\left(\frac{7}{2} ; \frac{k_{\perp}}{T_{c}}\right)\right],
\end{aligned}
$$

where $\Gamma$ is the incomplete gamma-function, $\tau$ is the proper time for plasma volume and $y$ is the photon rapidity, and $T_{c}=0.2 \mathrm{GeV}$ is the critical temperature at the end of the hydrodynamical evolution.

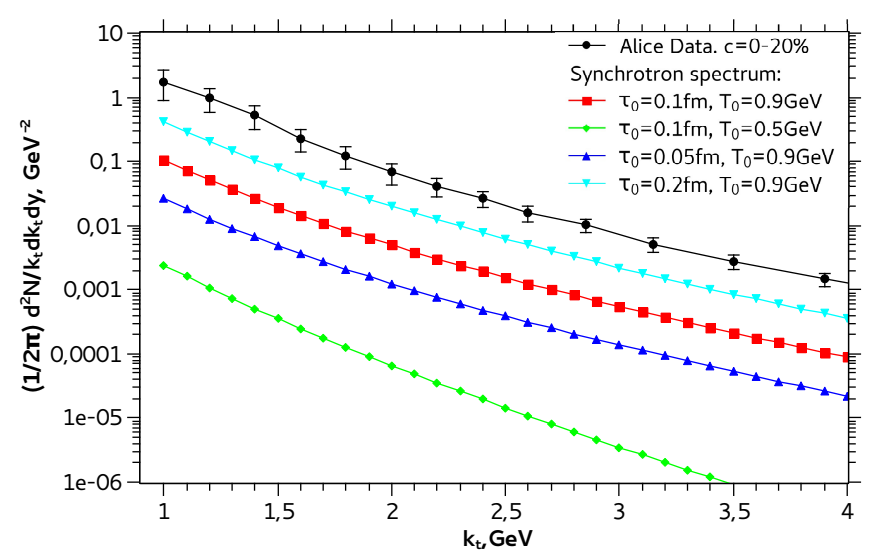

Figure 1: Spectra of synchrotron photons for different $T_{0}$ and $\tau_{0} . T_{0}$ is the temperature at the moment of proper time $\tau_{0}$, when the hydrodynamic regime begins. Experimental results taken from [10] for the centrality $0-20 \%$.

In Fig. 1 this spectrum (3.2) is presented for the different input parameters $T_{0}$ and $\tau_{0}$ at the transverse size of QGP system fixed as $r=10 \mathrm{fm}\left(T_{c}=0.2 \mathrm{GeV}\right.$ and $\sigma=0.2 \mathrm{GeV}^{2}$ are fixed). 
Experimental data from Ref. [10] in the most central $\mathrm{Pb}-\mathrm{Pb}$ collisions for the $0-20 \%$ centrality class at mid-rapidity $y$ in the transverse momentum range $1<k_{t}<4 \mathrm{GeV}$ are also given for comparison. In Fig. 2 we compare the spectrum (3.2) with experimental data for the 20-40 \% centrality class also and for this centrality class $<r(b)>\simeq 7 \mathrm{fm}$ (with $b$ being the impact parameter).

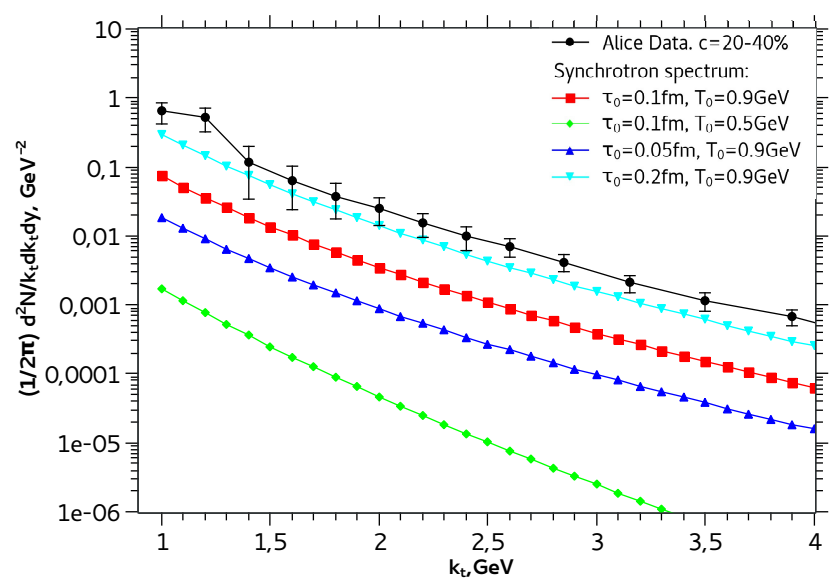

Figure 2: Spectra of synchrotron photons for different $T_{0}$ and $\tau_{0}$. Experimental results taken from [10] for the centrality $20-40 \%$.

\section{The peculiarities in angular distributions}

The synchrotron radiation is characterized by a high degree of photon polarization. The presence of a photon polarization is closely related to the geometrical feature of the QGP volume, over whose surface we should integrate.

In the picture of employing a hydrodynamical scaling solution, one has a cylindrically symmetric plasma volume (for central collisions) expanding in the longitudinal direction and the calculations for the final polarization can be done in explicit form. The primary degree of polarization

$$
P=\left(\frac{d N_{2}}{d \omega d t}-\frac{1}{2} \frac{d N_{\gamma}}{d \omega d t}\right) / \frac{d N_{\gamma}}{2 d \omega d t}=\frac{1}{2}
$$

reduces to about $20 \%$ for a plasma system. In Eq. (4.1) $N_{2}$ corresponds to linear photon polarization along the vector $\mathbf{e}_{2}$. The polarization vectors $\mathbf{e}_{1}$ and $\mathbf{e}_{2}$ are given by

$$
\mathbf{e}_{1}=\frac{\sigma \times \mathbf{k}}{|\sigma \times \mathbf{k}|}, \quad \mathbf{e}_{2}=\frac{\mathbf{k} \times \mathbf{e}_{1}}{\left|\mathbf{k} \times \mathbf{e}_{1}\right|},
$$

where $\mathbf{k}$ is the photon momentum.

After averaging these final photons (with a low degree of virtuality $\sqrt{k^{2}} \simeq \sqrt{\sigma}=0.45 \mathrm{GeV}$ ) are dominantly polarized along the normal to the plane spanned by the momentum of detected photons and the cylinder axis. Since the difficulties of registering photon polarization entail many problems for experimental search for this effect, we have recently suggested in Ref. [5] that observing leptonpair angular distribution resulting from the polarized photon conversion could be potentially an efficient probe of QGP. 
For the noncentral collisions the synchrotron radiation is nonisotropic and photons are emitted mainly around the direction determined by the normal to the ellipsoid-like surface (characterized by the eccentricity $\varepsilon$ ). This means that the photon azimuthal anisotropy, characterized by the second Fourier component:

$$
v_{2}^{\gamma}=\frac{\int d \phi_{\gamma} \cos \left(2 \phi_{\gamma}\right)\left(d N^{\gamma} / d \phi_{\gamma}\right)}{\int d \phi_{\gamma}\left(d N^{\gamma} / d \phi_{\gamma}\right)} \neq 0 \propto \varepsilon
$$

The coefficient of elliptic anisotropy for dilepton pairs will be also proportional to the eccentricity of QGP system as it takes place for the bremsstrahlung real photons and can be experimentally measured.

\section{Conclusions}

The main thesis of our present investigation is the following: the synchrotron radiation should be necessarily taken into consideration at a description of global photon and dilepton data in highenergy heavy-ion collisions. For the most central collisions the boundary photons contribute to the experimentally measured rate of direct photons at the level of $10 \%$.

To select this new uncommon mechanism of radiation unambiguously we suggest to study the noticeable specific anisotropy in the angle distribution of leptons with respect to the threemomentum of pair. The origin of this anisotropy is rooted in the existence of a characteristic direction in the field where the quarks are moving. Besides as another distinctive feature the synchrotron radiation will be nonisotropic for the noncentral collisions because the photons are dominantly emitted around the direction fixed by a surface normal, whose "mean" value is not zero.

Acknowledgments - The speaker would like to thank the Hard-Probes'18 organizers for the very generous welcome in Aix-Les-Bains. This work was supported by Russian Foundation for Basic Research (grant 18-02-00155).

\section{References}

[1] E.L. Feinberg, Nuovo Cimento A 34, 391 (1976).

[2] E.V. Shuryak, Yad Fiz. 28, 796 (1978) [Sov. J. Nucl. Phys. 28, 408 (1978)].

[3] J.-F. Paquet, J. Phys. Conf. Ser. 832 no.1, 012035 (2017).

[4] V.V. Goloviznin, A.M. Snigirev, and G.M. Zinovjev, JETP Lett. 98, 61 (2013).

[5] V.V. Goloviznin, A.M. Snigirev, and G.M. Zinovjev, JETP Lett. 107, 527 (2018).

[6] V.V. Goloviznin, A.V. Nikolskii, A.M. Snigirev, and G.M. Zinovjev, arXiv:1804.00559 [hep-ph].

[7] A. Casher, H. Neuberger, and S. Nussinov, Phys. Rev. D 20, 179 (1979).

[8] V.V. Goloviznin, A.M. Snigirev, and G.M. Zinovjev, Phys. Lett. B 211, 167 (1988).

[9] A.A. Sokolov and I.M. Ternov, Synchrotron Radiation [in Russian] (Nauka, Moscow, 1966).

[10] J. Adams et al. (ALICE collaboration), Phys. Lett. B 754, 235 (2016). 